\title{
Synchronous Price Discovery of Cross-Listings
}

\author{
Haiqiang Chen \\ Wang Yanan Institute for Studies in Economics, Xiamen University \\ Moon Sub Choi* \\ College of Business Administration, Ewha Womans University
}

(Received: November 11, 2013 / Revised: February 7, 2014 / Accepted: February 10, 2014)

\begin{abstract}
Extending from Grossman and Stiglitz (1980), we provide an asset pricing model of a synchronously traded crosslisted pair under information asymmetry. Following Garbade and Silber (1983), the model further embraces multimarket price discovery in a dynamic framework. The implications are as follows: The price sensitivity of holdings is higher for informed traders than for uninformed traders; the largest cross-border price spread occurs in the absence of arbitrageurs; price discovery is more likely in markets with a larger population of informed traders; and parity convergence accelerates with a higher price elasticity of demand of arbitrageurs.
\end{abstract}

Keywords: Price Discovery, Information Share, Informed Trading, Error Correction Model

* Corresponding Author, E-mail: paul.choi@ewha.ac.kr

\section{INTRODUCTION AND IMPLICATIONS}

Price discovery is a convergence process towards an equilibrium price (Schreiber and Schwartz, 1986) and is deemed one of the key elements of securities exchanges. It is the search mechanism by which information is reflected in asset valuation and return in the market. When a security trading is diversified (fragmented) in multiple venues, it is of scholastic import to detect where and how price discovery takes place. In the literature, Hasbrouck (1995) and Harris et al. (1995) study the exchange-respective relative contributions to the price discovery of fragmented stocks on the New York Stock Exchange (NYSE) and other U.S. regional exchanges and conclude that the NYSE assumes leadership. Regarding internationally cross-listed stocks, Bacidore and Sofianos (2002) and Solnik et al. (1996) argue that price discovery occurs dominantly on the home exchange due to its proximity to the locally incorporated issuers which generate price-affecting information. Eun and Sabherwal (2003) report that U.S. markets contribute to determining the prices of Canadian cross-listings but to a lower degree than the home Toronto Stock Exchange (TSX).
In our theoretical research, we extend the noisy rational expectations model of Grossman and Stiglitz (1980) in the following ways: First, a stock originally listed on TSX is cross-listed on near-integrated NYSE. Second, arbitrageurs are added to the model. Finally, we allow market friction. Our conclusion from the static model is that the price elasticity of demand by informed traders is higher than that of uninformed traders: An informed trader's confidence in the firm's future earnings is likely to render one's holdings more sensitive toward a dollar change in the price. The dollar premium on a cross-listing on NYSE over the original listing on TSX is the highest without arbitrageurs: It is negatively related to the demand elasticity of arbitrageurs and positively related to the transaction cost. Following Garbade and Silber (1983), our dynamic sequel embraces synchronous multi-market price discovery by deriving cointegration between the prices of a given cross-listed pair: Price discovery is more extensive in the market with a larger population of informed traders, and parity convergence accelerates with a higher price elasticity of demand of arbitrageurs.

In the rest of this paper, Sections 2 and 3 propose 
the aforementioned static and dynamic models.

\section{PRICE DISCOVERY OF A SYNCHRO- NOUSLY TRADED CROSS-LISTED PAIR}

For synchronous trades of the original listing and its cross-listing, we consider TSX and NYSE. ${ }^{1)}$ For convenience, we use $i=1,2$ to index these exchanges. There are three types of market participants: informed traders, uninformed traders, and arbitrageurs. Informed and uninformed traders are always engaged in trading, whereas arbitrageurs emerge only when there is an arbitrage opportunity. There are $N_{1}$ and $N_{2}$ market participants who trade only on TSX and NYSE, respectively, and $N_{3}$ arbitrageurs who trade on both exchanges.

Following Grossman and Stiglitz (1980), there are two types of one-market traders: informed versus uninformed traders with respective proportions in market $i$, $\pi_{i}$ and $\left(1-\pi_{i}\right)$. The future payoff $(v)$ of a risky asset (stock) is uncertain: $v \sim N\left(\bar{v}, \sigma_{v}^{2}\right)$. Informed traders recognize a signal $(S)$ about the payoff with noise: $\varepsilon_{s} \sim N\left(0, \sigma_{s}^{2}\right)$, where $S=v+\varepsilon_{s}$ and $\mathrm{E}\left(v \cdot \varepsilon_{s}\right)$. All variances are expressed in precision terms: $\tau_{v} \equiv 1 / \sigma_{v}^{2}$ and $\tau_{s} \equiv 1 / \sigma_{s}^{2}$. All traders in the two markets borrow and lend at the same risk-free rate $r$.

Trader $j$ in each market $i$ is initially endowed with shares, and the exchange-specific aggregate supply of shares is $Y_{i}$, where $\Delta Y_{i} \sim N\left(0, \sigma_{i}^{2}\right)$ is the random net supply from noise traders. All traders share the same constant absolute risk aversion (CARA) utility function with a risk aversion coefficient $(\rho)$ :

$$
V(W)=-e^{-\rho W}, \rho>0 .
$$

All random variables are assumed to be normally distributed, and so is wealth $(W)$. The investor's objective function is

$$
\mathrm{E}(W \mid \Phi)-(\rho / 2) \cdot \operatorname{Var}(W \mid \Phi),
$$

where $\Phi$ is the trader's information set.

The two markets are frictious with a quadratic transaction-cost function:

$$
C(x)=c x^{2} / 2,
$$

where $x$ is the number of stocks; and $c$ is the cofficient of marginal cost common to both exchanges.

\subsection{One-Market Traders}

Informed traders update their assumption of future payoffs based on private signals: An earnings surprise is

1) Exchange-rate market friction is considered neither in our static nor dynamic models unlike Grammig et al. (2005) due to the stationarity of the U.S.-Canada exchange rate (Issa et al., 2006).
$\Delta S \equiv S-\bar{S}$, where $\bar{S} \equiv \mathrm{E}(S)$ is the expected payoff. Prices of a given cross-listed pair are bullish on a positive earnings shock $(\Delta S>0)$. Informed traders in both markets, regardless of their locations, observe the same private signal $S$. Given a new earnings signal, their updated earnings forecast $(\mathrm{E}(v \mid S))$ and precision $(\tau(v \mid S))$ are given by

$$
\mathrm{E}(v \mid S)=\bar{v}+\left\{\tau_{s} /\left(\tau_{s}+\tau_{v}\right)\right\} \cdot \Delta S \text { and } \tau(v \mid S)=\tau_{s}+\tau_{v} .
$$

The demand from exchange-specific "homegeneous" informed trader $j\left(j=1,2, \cdots, N_{i}\right)$ for shares in market $i$ is

$$
\begin{aligned}
x_{i, j}^{I}\left(p_{i}, S\right) & =\left\{\mathrm{E}(v \mid S)-p_{i}(1+r)\right\} /\{\rho \operatorname{Var}(v \mid S)+c\} \\
& =\beta^{I}\left(\mu^{I}-p_{i}\right),
\end{aligned}
$$

where $\beta^{I} \equiv(1+r) /\left\{\rho /\left(\tau_{s}+\tau_{v}\right)+c\right\}$ is the elasticity of demand; $\mu^{I} \equiv\left[\bar{v}+\left\{\tau_{s} /\left(\tau_{s}+\tau_{v}\right)\right\} \cdot \Delta S\right] /(1+r)$ is the reservation price; and $p_{i}$ is the U.S. dollar-translated equilibrium price on exchange $i$, which is linear to the observables at any time:

$$
p_{i}=\alpha_{i 0}+\alpha_{i}^{S} \Delta S-\alpha_{i}^{Y} \Delta Y_{i}-\alpha_{-i}^{Y} \Delta Y_{-i},
$$

where $\alpha_{i}^{Y} \Delta Y_{i}$ and $\alpha_{-i}^{Y} \Delta Y_{-i}$ reflect reponses to supply shocks from its own ( $i$ ) and counterpart (-i) markets. ${ }^{2)}$

Uninformed traders observe prices on their respective exchanges and form their expectations of future earnings. $p_{1}$ and $p_{2}$ are the prices on TSX and NYSE, respectively, linear

The price function is "noisy" to uninformed traders due to supply shocks and earnings surprises. Uninformed traders extract information from prices only on their respective exchanges and their updated payoff forecast $\left(\mathrm{E}\left(v \mid p_{i}\right)\right)$ and precision $\left(\tau\left(v \mid p_{i}\right)\right)$ are

$$
\begin{aligned}
\mathrm{E}\left(v \mid p_{i}\right) & =\mathrm{E}(v)+\left\{\operatorname{Cov}\left(v, p_{i}\right) / \operatorname{Var}\left(p_{i}\right)\right\}\left\{p_{i}-\mathrm{E}\left(p_{i}\right)\right\} \\
& =\bar{v}+\left(\alpha_{i}^{S}\right)^{-1}\left\{\left(\phi_{i} \tau_{s}\right) /\left(\tau_{v}+\phi_{i} \tau_{s}\right)\right\} \Delta p_{i},
\end{aligned}
$$

where

$$
\begin{gathered}
\phi_{i} \equiv \frac{\tau_{1} \tau_{2}}{\tau_{1} \tau_{2}+h_{i 2}^{2} \tau_{2} \tau_{s}+h_{i 3}^{2} \tau_{1} \tau_{s}} \text { and } h_{i 1} \equiv \alpha_{i 1}^{Y} / \alpha_{i}^{s}, h_{i 2} \equiv \alpha_{i 2}^{Y} / \alpha_{i}^{s}, \\
\begin{array}{c}
\tau\left(v \mid p_{i}\right) \equiv\left\{\operatorname{Var}\left(v \mid p_{i}\right)\right\}^{-1}=\left\{\operatorname{Var}(v)-\operatorname{Cov}\left(v, p_{i}\right) 2 / \operatorname{Var}\left(p_{i}\right)\right\}^{-1} \\
=\tau_{v}+\frac{\tau_{1} \tau_{2} \tau_{s}}{\tau_{1} \tau_{2}+h_{i 2}^{2} \tau_{2} \tau_{s}+h_{i 3}^{2} \tau_{1} \tau_{s}}=\tau_{v}+\phi_{i} \tau_{s} .
\end{array}
\end{gathered}
$$

The demand function for uninformed traders is

$$
\begin{aligned}
x_{i}^{U}\left(p_{i}\right) & =\left[\left\{\mathbf{E}\left(v \mid p_{i}\right)-p_{i}(1+r)\right\} /\left\{\rho \mathbf{V a r}\left(v \mid p_{i}\right)+c\right\}\right] \\
& =\beta_{i}^{U}\left(\mu_{i}^{U}-p_{i}\right),
\end{aligned}
$$

2) For brevity, we use the notation $-i$ to denote the counterpart market: If $i=1$ (TSX), then $-i \equiv 2$ (NYSE). 
where

$$
\begin{aligned}
\beta_{i}^{U} & \equiv\left\{1+r-\frac{1}{\alpha_{i}^{s}}\left(\frac{\phi_{i} \tau_{s}}{\tau_{v}+\phi_{i} \tau_{s}}\right)\right\} /\left(\frac{\rho}{\tau_{v}+\phi_{i} \tau_{s}}+c\right), \\
\mu_{i}^{U} & \equiv\left\{\bar{v}-\frac{1}{\alpha_{i}^{s}}\left(\frac{\phi_{i} \tau_{s}}{\tau_{v}+\phi_{i} \tau_{s}}\right) \mathrm{E}\left(p_{i}\right)\right\} /\left\{1+r-\frac{1}{\alpha_{i}^{s}}\left(\frac{\phi_{i} \tau_{s}}{\tau_{v}+\phi_{i} \tau_{s}}\right)\right\} \\
& =\{\bar{v} /(1+r)\}-\lambda_{i}, \\
\lambda_{i} & \equiv\left(\alpha_{i 0}-\frac{\bar{v}}{1+r}\right)\left(\frac{1}{\alpha_{i}^{s}}\right)\left(\frac{\phi_{i} \tau_{s}}{\tau_{v}+\phi_{i} \tau_{s}}\right) /\left\{1+r-\frac{1}{\alpha_{i}^{s}}\left(\frac{\phi_{i} \tau_{s}}{\tau_{v}+\phi_{i} \tau_{s}}\right)\right\} .
\end{aligned}
$$

$\lambda_{i}$ can be shown to be zero in equilibrium, thus when the price is $p_{i}=\bar{v} /(1+r)$ uninformed traders will assume that there is no earnings surprise $(\Delta S=0)$ and they will demand no stock. Alternatively, if $p_{i}<(>) \bar{v} /(1+r)$, they will conjecture a negative (positive) signal from the observed price and choose to sell (buy) the stock. The following proposition is for the demand elasticity of informed and uninformed traders:

Proposition 1: The price elasticity of demand of informed traders is higher than that of uninformed traders: $\beta^{I}>\beta_{i}^{U}$ for $i=1,2$.

Proof: Since

$$
\begin{aligned}
& \beta_{i}^{U}=\left\{1+r-\frac{1}{\alpha_{i}^{s}}\left(\frac{\phi_{i} \tau_{s}}{\tau_{v}+\phi_{i} \tau_{s}}\right)\right\} /\left(\frac{\rho}{\tau_{v}+\phi_{i} \tau_{s}}+c\right), \\
& \beta^{I}=(1+r) /\left[\left\{\rho /\left(\tau_{s}+\tau_{v}\right)\right\}+c\right], \\
& \phi_{i}=\left(\tau_{1} \tau_{2}\right) /\left(\tau_{1} \tau_{2}+h_{i 2}^{2} \tau_{2} \tau_{s}+h_{i 3}^{2} \tau_{2} \tau_{s}\right), h_{i 2}=\alpha_{i 2} / \alpha_{i 1}, h_{i 3}=\alpha_{i 3} / \alpha_{i 1},
\end{aligned}
$$

we reach $\beta^{I}>\beta_{i}^{U}$ from $0<\phi_{i}<1$ and $1+r-\left(\alpha_{i 1}\right)^{-1}\left\{\left(\phi_{i} \tau_{s}\right)\right.$ $\left./\left(\tau_{v}+\phi_{i} \tau_{s}\right)\right\}<(1+r)$. Q.E.D.

\subsection{Arbitrageurs}

Arbitrageurs can go long and short in both markets. An arbitrageur holds a portfolio $\left(\left\{B, x_{1}^{A}, x_{2}^{A}\right\}\right)$ of $B$-many risk-free assets and $\left(x_{1}^{A}, x_{2}^{A}\right)$-many shares of a given cross-listed pair, subject to the initial wealth $\bar{B}$ :

$$
\bar{B}=B+p_{1} x_{1}^{A}+p_{2} x_{2}^{A}+c\left(x_{1}^{A}\right)^{2} / 2+c\left(x_{2}^{A}\right)^{2} / 2 .
$$

The future wealth is

$$
\begin{aligned}
W= & (1+r) B+v\left(x_{1}^{A}+x_{2}^{A}\right) \\
= & v\left(x_{1}^{A}+x_{2}^{A}\right) \\
& +(1+r)\left\{\bar{B}-p_{1} x_{1}^{A}-p_{2} x_{2}^{A}-c\left(x_{1}^{A}\right)^{2} / 2-c\left(x_{2}^{A}\right)^{2} / 2\right\} .
\end{aligned}
$$

Her objective function is

$$
\begin{aligned}
\mathrm{E}(W) & -(\rho / 2) \cdot \operatorname{Var}(W) \\
= & (1+r)\left\{\bar{B}-p_{1} x_{1}^{A}-p_{2} x_{2}^{A}-c\left(x_{1}^{A}\right)^{2} / 2-c\left(x_{2}^{A}\right)^{2} / 2\right\} \\
& +\mathrm{E}(v)\left(x_{1}^{A}+x_{2}^{A}\right)-(\rho / 2) \cdot \operatorname{Var}(v)\left(x_{1}^{A}+x_{2}^{A}\right)^{2} .
\end{aligned}
$$

She hedges perfectly to match their short position with long position: $x_{1}^{A}\left(p_{1}, p_{2}\right)+x_{2}^{A}\left(p_{1}, p_{2}\right)=0$. Accordingly, her demand function is

$$
x_{1}^{A}\left(p_{1}, p_{2}\right)=-x_{2}^{A}\left(p_{1}, p_{2}\right)=\left(p_{2}-p_{1}\right) /(2 c) .
$$

Arbitrageurs' aggregate demand is

$$
X_{1}^{A}\left(p_{1}, p_{2}\right)=-X_{2}^{A}\left(p_{1}, p_{2}\right)=N_{3} \cdot\left(p_{2}-p_{1}\right) /(2 c)=\beta^{A}\left(p_{2}-p_{1}\right),
$$

where $\beta^{A} \equiv N_{3} /(2 c)$ is their aggregate demand elasticity.

\subsection{Market equilibrium}

The market-clearing condition for each exchange is

$\sum_{j=1}^{N_{i} \pi_{i}} x_{i, j}^{I}\left(p_{i}, S\right)+\sum_{j=N_{i} \pi_{i}+1}^{N_{i}} x_{i, j}^{U}\left(p_{i}, S\right)+\beta^{A}\left(p_{-i}-p_{i}\right)=Y_{i}$.

Plugging in the demand functions for informed and uninformed traders gives:

$$
\pi_{i} N_{i} \beta^{I}\left(\mu^{I}-p_{i}\right)+\left(1-\pi_{i}\right) N_{i} \beta_{i}^{U}\left(\mu_{i}^{U}-p_{i}\right)+\beta^{A}\left(p_{-i}-p_{i}\right)=Y_{i} .
$$

Define

$$
\begin{aligned}
D_{i} & \equiv \beta^{I} \pi_{i} N_{i}+\beta_{i}^{U}\left(1-\pi_{i}\right) N_{i}, \\
\mu_{i} & \equiv\left\{\mu_{i}^{U} \beta_{i}^{U}\left(1-\pi_{i}\right) N_{i}+\mu^{I} \beta^{I} \pi_{i} N_{i}\right\} /\left\{\beta^{I} \pi_{i} N_{i}+\beta_{i}^{U}\left(1-\pi_{i}\right) N_{i}\right\},
\end{aligned}
$$

where $D_{i}$ is the exchange-specific aggregate demand elasticity; and $\mu_{i}$ is the weighted-average reservation price for informed and uninformed traders.

Since $\mu_{i}^{U}=\bar{v} /(1+r)-\lambda_{i}$ and $\mu^{I}=\left\{\bar{v}+\left(\frac{\tau_{s}}{\tau_{s}+\tau_{v}}\right) \Delta S\right\} /(1+r)$,

$$
\begin{aligned}
\mu_{i}=\frac{\bar{v}}{1+r} & +\Delta S \frac{1}{(1+r)} \frac{\tau_{s}}{\left(\tau_{s}+\tau_{v}\right)}\left\{\frac{\beta^{I} \pi_{i} N_{i}}{\beta^{I} \pi_{i} N_{i}+\beta_{i}^{U} N_{i}\left(1-\pi_{i}\right)}\right\} \\
& -\lambda_{i}\left[\left\{\beta_{i}^{U}\left(1-\pi_{i}\right) N_{i}\right\} /\left\{\beta^{I} \pi_{i} N_{i}+\beta_{i}^{U} N_{i}\left(1-\pi_{i}\right)\right\}\right] .
\end{aligned}
$$

The proportion of informed traders on exchange $i$ is

$$
\Pi_{i} \equiv\left(\beta^{I} \pi_{i} N_{i}\right) /\left\{\beta^{I} \pi_{i} N_{i}+\beta_{i}^{U} N_{i}\left(1-\pi_{i}\right)\right\} .
$$

Let $h \equiv \tau_{s} /\left(\tau_{s}+\tau_{v}\right)$ measure signal precision, then

$$
\mu_{i}=\left(\bar{v}+h \Pi_{i} \Delta S\right) /(1+r)-\lambda_{i}\left(1-\Pi_{i}\right) .
$$

The equilibrium price in market $i$ is

$$
p_{i}=\frac{\bar{v}}{1+r}-\frac{\lambda_{-i}\left(1-\Pi_{-i}\right) \beta^{A} / D_{i}+\lambda_{i}\left(1-\Pi_{i}\right)\left(1+\beta^{A} / D_{-i}\right)}{\left(1+\beta^{A} / D_{i}\right)+\beta^{A} / D_{-i}}
$$




$$
\begin{aligned}
& +\Delta S\left(\frac{h}{1+r}\right)\left\{\frac{\Pi_{-i} \beta^{A} / D_{i}+\Pi_{i}\left(1+\beta^{A} / D_{-i}\right)}{\left(1+\beta^{A} / D_{i}\right)+\beta^{A} / D_{-i}}\right\} \\
& -\frac{\left(\beta^{A}+D_{-i}\right) \Delta Y_{i}+\beta^{A} \Delta D_{-i}}{D_{-i} / D_{i}+\beta^{A}\left(D_{i}+D_{-i}\right)} .
\end{aligned}
$$

Comparing the equilibrium price with the linear conjecture model

$$
p_{i}=\alpha_{i 0}+\alpha_{i}^{S} \Delta S-\alpha_{i}^{Y} \Delta Y_{i}-\alpha_{-i}^{Y} \Delta Y_{-i}
$$

yields

$$
\begin{aligned}
\alpha_{i 0} & =\frac{\bar{v}}{1+r}-\frac{\lambda_{-i}\left(1-\Pi_{-i}\right) \beta^{A} / D_{i}+\lambda_{i}\left(1-\Pi_{i}\right)\left(1+\beta^{A} / D_{-i}\right)}{1+\beta^{A} / D_{i}+\beta^{A} / D_{-i}}, \\
\alpha_{i}^{S} & =\left(\frac{h}{1+r}\right)\left\{\frac{\Pi_{-i} D_{-i} \beta^{A}+D_{i} \Pi_{i}\left(D_{-i}+\beta^{A}\right)}{D_{-i} D_{i}+\beta^{A}\left(D_{i}+D_{-i}\right)}\right\}, \\
\alpha_{i}^{Y} & =\left(\beta^{A}+D_{-i}\right) /\left\{D_{-i} D_{i}+\beta^{A}\left(D_{i}+D_{-i}\right)\right\}, \\
\alpha_{-i}^{Y} & =\beta^{A} /\left\{D_{-i} D_{i}+\beta^{A}\left(D_{i}+D_{-i}\right)\right\} .
\end{aligned}
$$

Since

$$
\lambda_{i}=\left\{\alpha_{i 0}-\bar{v} /(1+r)\right\} \theta_{i}
$$

where

$$
\theta_{i}=\left(\frac{1}{\alpha_{i 1}}\right)\left(\frac{\phi_{i} \tau_{s}}{\tau_{v}+\phi_{i} \tau_{s}}\right) /\left\{1+r-\frac{1}{\alpha_{i 1}}\left(\frac{\phi_{i} \tau_{s}}{\tau_{v}+\phi_{i} \tau_{s}}\right)\right\},
$$

we have

$$
\lambda_{i}=\left[\frac{\left\{\lambda_{-i}\left(1-\Pi_{-i}\right)\right\}\left(1+\beta^{A} / D_{i}\right)+\left\{\lambda_{i}\left(1-\Pi_{i}\right)\right\}\left(1+\beta^{A} / D_{-i}\right)}{1+\beta^{A} / D_{i}+\beta^{A} / D_{-i}}\right] \theta_{i} .
$$

In equilibrium $\lambda_{1}=\lambda_{2}=0$, thus it leads to

$$
\mu_{i}=\left(\bar{v}+h \Pi_{i} \Delta S\right) /(1+r),
$$

which is the market-expected price of the listing on exchange $i$. When the signal is more informative $\left(\Pi_{i}\right)$, we have a higher (lower) market-expected value for a positive (negative) signal.

The dollar premium on the cross-listing on NYSE over the original listing on TSX is

$$
\begin{aligned}
p_{2}-p_{1}= & \Delta S\left(\frac{h}{1+r}\right)\left(\frac{\Pi_{2}-\Pi_{1}}{1+\beta^{A} / D_{1}+\beta^{A} / D_{2}}\right) \\
& +\frac{\Delta Y_{1} / D_{1}-\Delta Y_{2} / D_{2}}{1+\beta^{A} / D_{1}+\beta^{A} / D_{2}}
\end{aligned}
$$

and it varies over the range of $\beta^{A}$ :

Case I: $\beta^{A}=\infty$. In perfect markets with no transaction cost $(c=0)$, arbitrageurs have an infinite demand elasticity leading to $p_{2}-p_{1}=0$, thus the efficient market price $\left(p_{e}\right)$ is

$$
p_{1}-p_{2}=p_{e} \equiv \frac{\bar{v}}{1+r}+\Delta S\left(\frac{h}{1+r}\right)\left\{\frac{\Pi_{2} D_{2}+\Pi_{1} D_{1}}{D_{1}+D_{2}}\right\}-\frac{\Delta Y_{1}+\Delta Y_{2}}{\left(D_{1}+D_{2}\right)} .
$$

Case II: $0<\beta^{A}<\infty$. With limits to arbitrage,

$$
\begin{aligned}
p_{2}-p_{1}= & \Delta S\left(\frac{h}{1+r}\right)\left(\frac{\Pi_{2}-\Pi_{1}}{1+\beta^{A} / D_{1}+\beta^{A} / D_{2}}\right) \\
& +\frac{\Delta Y_{1} / D_{1}+\Delta Y_{2} / D_{2}}{1+\beta^{A} / D_{1}+\beta^{A} / D_{2}} .
\end{aligned}
$$

Case III: $\beta^{A}=\infty$. In the absense of arbitrage,

$$
p_{2}-p_{1}=\Delta S\{h /(1+r)\}\left(\Pi_{2}-\Pi_{1}\right)+\Delta Y_{1} / D_{1}-\Delta Y_{2} / D_{2} .
$$

In conclusion, the dollar premium on the cross-listing over its original listing is the highest in the absence of arbitrageurs. The price spread is negatively related to the demand elasticity of arbitrageurs and positively related to the transaction cost, consistent with the empirical findings of Gagnon and Karolyi (2010).

\section{PRICE DISCOVERY AND ERROR CORRECTION DYNAMICS}

The model presented in Section 2 describes the static equilibrium relationship between the two prices of a given cross-listed pair. However, in the time series context the equilibrium model must account for the evolution of market reservation prices $\mu_{i, t}(i=1,2)$. Following Garbade and Silber (1983) and Kyle (1985), the dynamics of $\mu_{i, t}$ is

$$
\mu_{i, t}=\mu_{i, t-1}+h \Pi_{i} \Delta S_{t}+\varepsilon_{i t},
$$

where $\Delta S_{t}$ reflects the arrival of a new information signal between periods $t-1$ and $t ; \Delta S_{t}$ and $\varepsilon_{i t}$ are assumed to be stationary processes; and $\mu_{i, t}$ follows a random walk. Moreover, after the market clearance at the end of the period $t-1, p_{i t-1}$ is the reservation price for every trader in market $i$, thus

$$
\mu_{i, t}=p_{i, t-1}+h \Pi_{i} \Delta S_{t}+\varepsilon_{i t} .
$$

The equilibrium price from Section 2 is

$$
p_{i, t}=\frac{\mu_{-i, t}\left(\beta^{A} / D_{i}\right)+\mu_{i, t}\left(\beta^{A} / D_{-i}\right)}{\left(1+\beta^{A} / D_{i}\right)+\beta^{A} / D_{-i}}-\frac{\left(\beta^{A} / D_{-i}\right) \Delta Y_{i t}+\beta^{A} \Delta Y_{-i t}}{D_{-i} D_{i}+\beta^{A}\left(D_{i}+D_{-i}\right)} .
$$


By plugging the reservation prices in the equilibrium price functions:

$$
\left(\begin{array}{l}
p_{1, t} \\
p_{2, t}
\end{array}\right)=\left(\begin{array}{l}
1-a, a \\
b, 1-b
\end{array}\right)\left(\begin{array}{l}
p_{1, t-1} \\
p_{2, t-1}
\end{array}\right)+\left(\begin{array}{c}
1-a, a \\
b, 1-b
\end{array}\right)\left(\begin{array}{c}
\Delta Y_{1 t} / D_{1}+h \Pi_{1} \Delta S_{t}+\varepsilon_{1 t} \\
\Delta Y_{2 t} / D_{2}+h \Pi_{2} \Delta S_{t}+\varepsilon_{2 t}
\end{array}\right),
$$

where

$$
\begin{aligned}
& a=\left(\beta^{A} / D_{1}\right) /\left(1+\beta^{A} / D_{1}+\beta^{A} / D_{2}\right), \\
& b=\left(\beta^{A} / D_{2}\right) /\left(1+\beta^{A} / D_{1}+\beta^{A} / D_{2}\right) .
\end{aligned}
$$

A resulting vector error correction model (VECM) can be derived by subtracting $\left(p_{1, t-1}, p_{2, t-1}\right)^{T}$ from both sides:

$$
\left(\begin{array}{l}
\Delta p_{1, t} \\
\Delta p_{2, t}
\end{array}\right)=\left(\begin{array}{c}
-a, a \\
b,-b
\end{array}\right)\left(\begin{array}{l}
p_{1, t-1} \\
p_{2, t-1}
\end{array}\right)+\left(\begin{array}{l}
1-a, a \\
b, 1-b
\end{array}\right)\left(\begin{array}{l}
\varepsilon_{1 t} \\
\varepsilon_{2 t}
\end{array}\right) .
$$

When $\beta^{A} \neq 0$, we can apply permanent-transitory decomposition (Gonzalo and Granger, 1995) to the above VECM. The permanent component reflects a linear combination of the two prices $\left(p_{1, t}, p_{2, t}\right)$ formed by the scaled orthogonal vector of the adjustment coefficient vector $(a, b)$ :

$$
f_{t}=\{b /(a+b)\} p_{1, t}+\{a /(a+b)\} p_{2, t} .
$$

Since

$$
\begin{aligned}
& \frac{b}{a+b}=\frac{\beta^{A} /\left\{\beta_{2}^{U} N_{2}\left(1-\pi_{2}\right)+\beta^{I} \pi_{2} N_{2}\right\}}{\sum_{i=1}^{2}\left[\beta^{A} / \beta_{i}^{U} N_{i}\left(1-\pi_{i}\right)+\beta^{I} \pi_{i} N_{i}\right]} \\
& =\frac{\beta^{I} \pi_{1} N_{1}+\beta_{1}^{U} N_{1}\left(1-\pi_{1}\right)}{\sum_{i=1}^{2}\left[\beta^{I} \pi_{i} N_{i}+\beta_{i}^{U} N_{i}\left(1-\pi_{i}\right)\right]} \\
& \left.\quad=\left\{D_{1} / D_{1}+D_{2}\right)\right\}, \\
& a /(a+b)=D_{2} /\left(D_{1}+D_{2}\right),
\end{aligned}
$$

we have

$$
f_{t}=\frac{\bar{t}_{t}}{1+r}+\Delta S_{t}\left(\frac{h}{1+r}\right)\left\{\frac{\sum_{i=1}^{2}\left(\Pi_{i} D_{i}\right)}{\sum_{i=1}^{2} D_{i}}\right\}-\frac{\sum_{i=1}^{2} \Delta Y_{i t}}{\sum_{i=1}^{2} D_{i}} \equiv p_{t}^{e} .
$$

Note that the derived permanent component $\left(p_{t}^{e}\right)$ is the price under the condition that $\beta^{A} \rightarrow \infty$ (perfect market). The information share of exchange $i$,

$$
I S_{i} \equiv D_{i} /\left(D_{1}+D_{2}\right),
$$

reflects the relative contribution share of each exchangespecific price to the permanent component. Hasbrouck (1995) and Figuerola-Ferretti and Gonzalo (2009) find $b=N_{1}$ and $a=N_{2}$ and thus view the information share as the relative number of participants in respective market. However, they assume that all market participants are informed $\left(\pi_{1}=\pi_{2}=1\right)$, which is a special case of our following proposition:

Proposition 2: Price discovery is more likely in the market with a higher proportions of informed traders and more market partipants, that is, in markets with a larger population of informed traders: Given $\Pi_{i}>\Pi_{-i}$ and $N_{i}$ $\geq N_{-i}$, we have $I S_{i}>I S_{-i}$,

Proof: From Proposition 1, $\beta^{I}>\beta_{i}^{U}$ for all $i=1$, 2. Since $\left.D_{i} \equiv \beta^{I} \pi_{i} N_{1}+\beta_{i}^{U}\left(1-\pi_{i}\right) N_{i}, \Pi_{i}=\left\{\beta^{I} \pi_{i} N_{i}\right) / D_{i}\right\}$. It follows that $D_{i}>D_{-i}$, thus $I S_{i}>I S_{-i}$. Q.E.D.

The above prediction provides support for Eun and Sabherwal (2003): The information share of NYSE is related to trading volume.

Finally, we discuss the rate of the convergence of the two market prices to their long-run equilibrium. We conjecture a first-order autoregressive (AR(1)) model by using the price spread, and the first-order coefficient is taken as the rate of convergence to equilibrium parity:

$$
\Delta p_{t}=\delta \Delta p_{t-1}+\varepsilon_{t},
$$

where (1) $\Delta p_{t}=p_{2 t}-p_{1 t}$ is the sychronous dollar pre-mium on the cross-listing; (2) $\delta$ is the rate of conver-gence:

$$
\delta=1-a-b=\left\{1+\sum_{i=1}^{2}\left(\beta^{A} / D_{i}\right)\right\}^{-1} .
$$

The proposition for the rate of convergence is provided without proof:

Proposition 3: The price elasticity of demand of arbitrageurs $\beta^{A}$ has no direct relationship with the information share of each market, but it is positively related to the short-term convergence rate $\delta$.

In other words, parity convergence accelerates when arbitrageurs become more sensitive to dollar changes in the price.

\section{ACKNOWLEDGEMENT}

We thank Jaewook Lee (Editor) and referees for their invaluable comments and feedback. Specials thanks are due to Warren B. Bailey, Yongmiao Hong, G. Andrew Karolyi, and Maureen O'Hara for their teachings. This work was supported by the National Research Foundation of Korea Grant funded by the Korean Government (NRF-2011-332-B00189). Standard disclaimer rules apply and all errors are of our own. 


\section{REFERENCES}

Eun, C. and S. Sabherwal, "Cross-border listings and price discovery: Evidence from U.S.-listed Canadian stocks," Journal of Finance 58 (2003), 549574.

Figuerola-Ferretti, I. and J. Gonzalo, "Modeling and measuring price discovery in commodity markets," Journal of Econometrics 158 (2010), 95-107.

Gagnon, L. and G. A. Karolyi, "Multi-Market trading and arbitrage," Journal of Financial Economics 97 (2010), 53-80.

Garbade, K. D. and W. L. Silber, "Price movements and price discovery in futures and cash markets," $R e$ view of Economics and Statistics 65 (1983), 289297.

Gonzalo, J. and C. W. J. Granger, "Estimation of common long-memory components in cointegrated sys- tems," Journal of Business and Economic Statistics 13 (1995), 1-9.

Grammig, J., M. Melvin, and C. Schlag, "Internationally cross-listed stock prices during overlapping trading hours: price discovery and exchange rate effects," Journal of Empirical Finance 12 (2005), 139-164.

Grossman, S. and J. Stiglitz, "On the impossibility of informationally efficient markets," American Economic Review 70 (1980), 393-408.

Hasbrouck, J., "One security, many markets: determining the contributions to price discovery," Journal of Finance 50 (1995), 1175-1201.

Issa, R., R. Lafrance, and J. Murray, The turning black tide: energy prices and the Canadian dollar, Working Paper, Bank of Canada, 2006.

Kyle, A., "Continuous auctions and insider trading," Econometrica 53 (1985), 1315-1335. 